\title{
On the existence of limit cycles for some planar vector fields
}

\author{
L. Rocío GonzÁlez-Ramírez ${ }^{a, b}$ Osvaldo Osuna $^{a *}$, \\ Rubén SAntaella-Forero ${ }^{a}$ \\ ${ }^{a}$ Universidad Michoacana de San Nicolás de Hidalgo, Instituto de Física y \\ Matemáticas, Michoacán, México. \\ ${ }^{b}$ Catedrática Conacyt.
}

\begin{abstract}
In this work, we prove the existence of limit cycles in planar systems that can be written as appropriate perturbations of Hamiltonian systems. In particular, we obtain criteria for the existence of limit cycles for Liénard-type systems. We present examples in order to illustrate our results. Keywords: Poincaré-Bendixson theorem, trapping region, Liénard equation, limit cycles.
\end{abstract}

MSC2010: 34C07, 34C05, 34C25.

\section{Sobre la existencia de ciclos límite de ciertos campos vectoriales en el plano}

\begin{abstract}
Resumen. En este trabajo, demostramos la existencia de ciclos límite en sistemas planos que pueden escribirse como perturbaciones apropiadas de sistemas Hamiltonianos. En particular, obtenemos criterios de existencia de ciclos límite para sistemas tipo Liénard. Además, presentamos algunos ejemplos con el fin de ilustrar los resultados obtenidos.

Palabras clave: Teorema de Poincaré-Bendixson, anillo invariante, ecuación de Liénard, ciclos límite.
\end{abstract}

\section{Introduction}

The main goal of the qualitative theory of differential equations is the topological description of properties and configurations of solutions of differential systems in the whole space or the subset wherein they are defined. In the planar case, the periodic

*E-mail: osvaldo@ifm.umich.mx

Received: 9 July 2015, Accepted: 24 November 2015.

To cite this article: L.R. González-Ramírez, O. Osuna, R. Santaella-Forero, On the existence of limit cycles

for some planar vector fields, Rev. Integr. Temas Mat. 33 (2015), No.2, 191-198. 
orbits (in particular, the limit cycles) are one of the most important objects in the study of differential systems. Given an open set $\Omega \subseteq \mathbb{R}^{2}$, we consider the system

$$
\left\{\begin{array}{l}
\dot{x}=f_{1}(x, y), \\
\dot{y}=f_{2}(x, y), \quad(x, y) \in \Omega,
\end{array}\right.
$$

where $f_{1}$ and $f_{2}$ are real $C^{1}$-functions on $\Omega$. Considering the vector field $F(x, y)=$ $\left(f_{1}(x, y), f_{2}(x, y)\right)$, the system (1) can be rewritten in the form $\dot{w}=F(w), w=(x, y) \in$ $\Omega$. An important class of trajectories of (1) are given by the limit cycles.

Definition 1.1. A limit cycle of the system (1) is a periodic orbit $\gamma \in \Omega$ for which there is at least one other solution tending towards $\gamma$ when $t \rightarrow+\infty$ or $t \rightarrow-\infty$.

It is well known that for a polynomial vector field a limit cycle is a periodic orbit which has an annulus-like neighborhood free of other periodic solutions.

The Poincaré-Bendixson theorem (see [2],[6] and [5]) plays an important role in the study of the qualitative behaviour of autonomous differential equations on $\mathbb{R}^{2}$. It describes the structure of limit sets in such systems. An immediate and useful consequence of PoincaréBendixson theorem which is often applied to prove the existence of limit cycles is the following:

Corollary 1.2. If the system (1) has a positively (negatively) invariant annular region $A \subset \Omega$ that is singular-point free, then $A$ contains at least one periodic orbit. If in addition, some point in the interior of $A$ is in the forward (backward) orbit of a point on the boundary of $A$, then $A$ contains at least one limit cycle.

Of course, the main difficulty in applying the above result is to find a suitable annular region $A$ (also called a trapping region). A classic strategy to obtain trapping regions is to study the behavior of the vector field on the boundary of domains delimited by the level sets of an adequate real function (see [1],[3],[5] and [7]), we take advantage of this argument for our results.

This work deals with the problem of existence of limit cycles for some planar differential systems, written as convenient perturbations of Hamiltonian systems. We provide a method to construct vector fields admitting trapping regions. In particular, we obtain criteria for the existence of limit cycles for Liénard-type systems. We also present several examples to illustrate our results.

\section{Existence Results}

Given $H$ a $C^{2}$-function defined on $\mathbb{R}^{2}$, we consider the following assumptions on it:

(H1) $H^{-1}(0)=\{(0,0)\}$.

(H2) There exists $c_{\max }$, such that $H^{-1}(c)$ delimits the boundary of a convex region for all $0<c<c_{\max }$, with the possibility of having $c_{\max }=\infty$.

As usual, the Hamiltonian vector field $X_{H}$ is defined by $X_{H}:=\left(\frac{\partial H}{\partial y},-\frac{\partial H}{\partial x}\right)$.

Definition 2.1. Assume that $H: \mathbb{R}^{2} \rightarrow \mathbb{R}$ satisfies (H1)-(H2), and let $f: \mathbb{R}^{2} \rightarrow \mathbb{R}$ be a $C^{1}$-function. We say that $f$ is " $H$-change sign", if the following conditions hold: 
i) $f(0,0)<0($ or $f(0,0)>0)$;

ii) there is a real number $r_{f}>0$, with $r_{f}<c_{\max }$ such that $f(x, y)>0(f(x, y)<0$, respectively) in $\left\{(x, y) \in \mathbb{R}^{2} \mid H(x, y) \geq r_{f}\right\}$.

We say that $f_{1}, f_{2}$ are $H$-change sign of the same type if $f_{1}(0,0)$ and $f_{2}(0,0)$ have the same sign.

Theorem 2.2. Assume that $H: \mathbb{R}^{2} \rightarrow \mathbb{R}$ satisfies (H1)-(H2) and the origin is the only critical point of $X_{H}$. Let $f, g: \mathbb{R}^{2} \rightarrow \mathbb{R}$ be $C^{1}$-functions. Suppose that $f$ is $H$-change sign and that $g$ nowhere vanishes. Then, the vector field

$$
X:=g X_{H}+\left(0,-f(x, y) \frac{\partial H}{\partial y}\right)
$$

admits at least one limit cycle.

Proof. Assume the case $f(0,0)<0$. Since the origin is the only singular point of $X_{H}$, then it is the only singular point of the vector field $X$. Consider the level curves $H_{c}=$ $\left\{(x, y) \in \mathbb{R}^{2} \mid H(x, y)=c\right\}$, for $0<c<c_{\max }$. Consider $\alpha_{c}$ to be the angle between $H_{c}$ and the vector field $X$. This angle is determined by the inner product of $\nabla H$ and $X$ evaluated at $H_{c}$, obtaining:

$$
\alpha_{c}=g \frac{\partial H}{\partial x} \frac{\partial H}{\partial y}-g \frac{\partial H}{\partial y} \frac{\partial H}{\partial x}-f(x, y)\left(\frac{\partial H}{\partial y}\right)^{2}=-f(x, y)\left(\frac{\partial H}{\partial y}\right)^{2} .
$$

Given the assumptions on $f(x, y)$, we have that close to the origin trajectories of $X$ point outward to the level curves of $H$, and far away from the origin (when $H(x, y) \geq r_{f}$ ) trajectories point inward. Using Poincaré-Bendixson theorem we conclude that the trapping region must contain at least one limit cycle.

From Theorem 2.2 we require that the origin is the only singular point of the Hamiltonian system. The following easy result gives some sufficient conditions for uniqueness of singular point.

Lemma 2.3. Let $H: \mathbb{R}^{2} \rightarrow \mathbb{R}$ be a $C^{2}$-function; then the origin is the only singular point of $X_{H}$, if any of the following conditions holds:

- $y \frac{\partial H}{\partial y}(0, y)>0$ for $y \neq 0$;

- $y_{1} \geq y_{2}$ for $x_{1}<x_{2}$ where $\frac{\partial H}{\partial y}\left(x_{i}, y_{i}\right)=0$ for $i=1,2$;

- $x \frac{\partial H}{\partial x}(x, 0)>0$ for $x \neq 0$;

- $y_{1} \leq y_{2}$ for $x_{1}<x_{2}$ where $\frac{\partial H}{\partial x}\left(x_{i}, y_{i}\right)=0$ for $i=1,2$.

- $y \frac{\partial H}{\partial y}(0, y)>0$ for $y \neq 0$;

- $y_{1} \leq y_{2}$ for $x_{1}<x_{2}$ where $\frac{\partial H}{\partial y}\left(x_{i}, y_{i}\right)=0$ for $i=1,2$;

- $x \frac{\partial H}{\partial x}(x, 0)>0$ for $x \neq 0$;

Vol. 33, No. 2, 2015] 
- $y_{1} \geq y_{2}$ for $x_{1}<x_{2}$ where $\frac{\partial H}{\partial x}\left(x_{i}, y_{i}\right)=0$ for $i=1,2$.

- The origin is the unique critical point of the function $H$.

(V4) - The function $H$ is positive definite.

Remark 2.4. Furthermore, in the assumptions (V1) and (V2) we guarantee that the trajectories turn clockwise.

Proposition 2.5. Assume that $H: \mathbb{R}^{2} \rightarrow \mathbb{R}$ satisfies (H1)-(H2) and that (V1), (V2), (V3) or (V4) hold true. Let $f, g: \mathbb{R}^{2} \rightarrow \mathbb{R}$ be $C^{1}$-functions. Suppose that $f$ is $H$-change sign and that $g$ nowhere vanishes. Then the vector field

$$
X:=g X_{H}+\left(-f(x, y) \frac{\partial H}{\partial x}, 0\right)
$$

admits at least one limit cycle.

Proof. The proof is similar to Theorem 2.2 and we omit it.

Corollary 2.6. Under the hypotheses of Theorem 2.2 and $n \in \mathbb{N} \cup\{0\}$, the vector fields
i) $X:=g X_{H}+\left(-f(x, y)\left(\frac{\partial H}{\partial x}\right)^{2 n+1}, 0\right)$,
ii) $Y:=g X_{H}+\left(0,-f(x, y)\left(\frac{\partial H}{\partial y}\right)^{2 n+1}\right)$,

admit at least one limit cycle.

Let us consider the following Liénard-type system:

$$
\left\{\begin{array}{l}
\dot{x}=y^{2 n+1} \\
\dot{y}=-x^{2 m+1}-f(x, y) y^{2 n+1}
\end{array}\right.
$$

where $m, n \in \mathbb{N} \cup\{0\}$. We obtain the next result:

Corollary 2.7. Let $f: \mathbb{R}^{2} \rightarrow \mathbb{R}$ be a $C^{1}$-function, and suppose that the following conditions hold:

i) $f(0,0)<0($ or $f(0,0)>0)$;

ii) there is $r_{0}$ such that $f(x, y)>0\left(f(x, y)<0\right.$ respectively) for all $(x, y) \in \mathbb{R}^{2}$ with $x^{2 m+2}+y^{2 n+2} \geq r_{0}$.

Then the system (4) admits at least one limit cycle.

Proof. Taking $H(x, y)=\frac{x^{2 m+2}}{2 m+2}+\frac{y^{2 n+2}}{2 n+2}$ and $g=1$, then the vector field (2) yields the system (4), thus the result follows from Theorem 2.2. 
Proposition 2.8. Assume that $H: \mathbb{R}^{2} \rightarrow \mathbb{R}$ satisfies (H1)-(H2) and the origin is the only critical point of $X_{H}$. Let $f_{i}: \mathbb{R}^{2} \rightarrow \mathbb{R}, i=1, \ldots, j$, for $j \in \mathbb{N}, j<\infty$ be $C^{1}$-functions. Suppose that the functions $f_{i}$ are $H$-change sign of the same type and $n_{i} \in \mathbb{N} \cup\{0\}$. Then the vector field

$$
X:=X_{H}-\left(0, \sum_{i=1}^{j} f_{i}\left(\frac{\partial H}{\partial y}\right)^{2 n_{i}+1}\right)
$$

admits at least one limit cycle.

Proof. The proof is analogous to that of Theorem 2.2 and we omit it.

A slight variation of the above proposition is given by the following

Proposition 2.9. Assume that $H: \mathbb{R}^{2} \rightarrow \mathbb{R}$ satisfies (H1)-(H2) and that (V1), (V2), (V3) or (V4) hold true. Let $f_{i}: \mathbb{R}^{2} \rightarrow \mathbb{R}, i=1,2$ be $C^{1}$-functions. Suppose that $f_{1}$ is $H$-change sign, $f_{1}(0,0)<0, f_{2} \geq 0$ and $n \in \mathbb{N}$. Then the vector field

$$
X:=X_{H}-\left(0, f_{1}\left(\frac{\partial H}{\partial y}\right)+f_{2}\left(\frac{\partial H}{\partial y}\right)^{2 n+1}\right)
$$

admits at least one limit cycle.

\section{Examples and applications}

We now explore examples of planar systems, written as convenient perturbations of Hamiltonian systems. Using our previous results we prove the existence of at least one limit cycle in such systems.

Example 3.1. Consider $H(x, y)=2 x^{2}+2 x y+y^{2}$ a positive definite function and $f(x, y)=$ $x^{2}+2 y^{2}-2$. Then the system

$$
\left\{\begin{array}{l}
\dot{x}=2 x+2 y \\
\dot{y}=-4 x-2 y-\left(x^{2}+2 y^{2}-2\right)(2 x+2 y)
\end{array}\right.
$$

admits at least one limit cycle by Theorem 2.2.

Example 3.2. Consider $H(x, y)=x^{4}+x^{2}+y^{4}$ and $f(x, y)=2 x^{2}+y^{2}-1$. Then the system

$$
\left\{\begin{array}{l}
\dot{x}=4 y^{3}, \\
\dot{y}=-4 x^{3}-2 x-4\left(2 x^{2}+y^{2}-1\right) y^{3},
\end{array}\right.
$$

admits at least one limit cycle by Theorem 2.2.

Vol. 33, No. 2, 2015] 


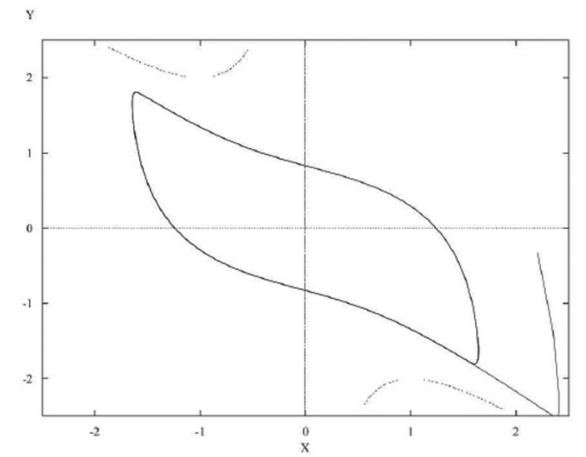

Figure 1. Example 3.1.

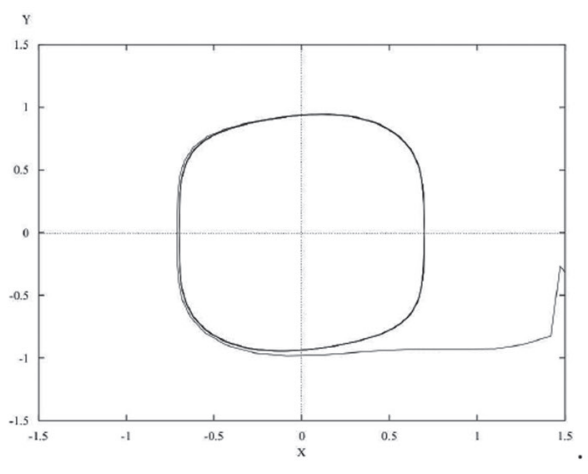

Figure 2. Example 3.2.

Example 3.3. For $H(x, y)=x^{2}+x y+y^{2}$ and $f(x, y)=x^{2}+y^{4}-1$, we consider

$$
\left\{\begin{array}{l}
\dot{x}=2 y+x \\
\dot{y}=-2 x-y-\left(x^{2}+y^{4}-1\right)(2 y+x)^{3} .
\end{array}\right.
$$

By Corollary 2.6 the system admits at least one limit cycle.

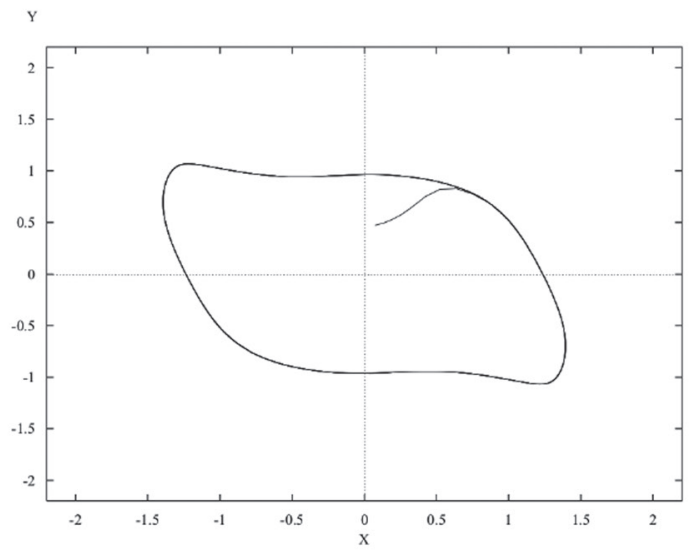

Figure 3. Example 3.3.

Example 3.4. Assume that $H: \mathbb{R}^{2} \rightarrow \mathbb{R}$ satisfies (H1)-(H2) and that the origin is the only singular point of $X_{H}$. Let $\alpha: \mathbb{R} \rightarrow \mathbb{R}$ be nonnegative, monotone increasing (resp. decreasing) for $x>0$ (resp. $x<0$ ) continuous function and $\alpha(0)<r<\sup \alpha(x)$. Then the vector field

$$
X:=a X_{H}+\left(0,-(\alpha(H)-r) \frac{\partial H}{\partial y}\right), a \neq 0, \alpha(0)<r<\sup \alpha(H),
$$

admits at least one limit cycle. 
The next corollary recovers the existence part of Corollary 3.1.1 in [4]:

Corollary 3.5. Let us consider system

$$
\left\{\begin{array}{l}
\dot{x}=y^{2 n+1}, \\
\dot{y}=-x^{2 n+1}-f(x, y) y^{2 n+1}, n \in \mathbb{N} \cup\{0\},
\end{array}\right.
$$

and let $f: \mathbb{R}^{2} \rightarrow \mathbb{R}$ be a $C^{1}$-function, and suppose that the following conditions hold:

i) $f(0,0)<0($ or $f(0,0)>0)$;

ii) there is $r_{0}$ such that $f(x, y)>0\left(f(x, y)<0\right.$ respectively) for all $(x, y) \in \mathbb{R}^{2}$ with $x^{2 n+2}+y^{2 n+2} \geq r_{0}$.

Then the system (7) admits at least one limit cycle.

This can be seen by using $H(x, y)=\frac{1}{2 n+2}\left(x^{2 n+2}+y^{2 n+2}\right)$. We also note that the preceding corollary includes some results in [3], Section 4.

Example 3.6. Taking $H(x, y)=x^{2}+x^{4}+y^{2}$, we consider the system

$$
\left\{\begin{array}{l}
\dot{x}=2 y, \\
\dot{y}=4 x^{3}-2 x-\left(x^{2}+y^{4}-1\right)(2 y)-\left(x^{4}+y^{2}-1\right)(2 y)^{3} .
\end{array}\right.
$$

By Proposition 2.8, the system admits at least one limit cycle.

Example 3.7. Taking $H(x, y)=e^{x^{4}+y^{4}}$, we consider the system

$$
\left\{\begin{array}{l}
\dot{x}=4 y^{3} e^{x^{4}+y^{4}}, \\
\dot{y}=4 x^{3} e^{x^{4}+y^{4}}-\left(x^{2}+y^{2}+y^{4}-1\right)\left(4 y^{3} e^{x^{4}+y^{4}}\right) .
\end{array}\right.
$$

By Theorem 2.2, the system admits at least one limit cycle.

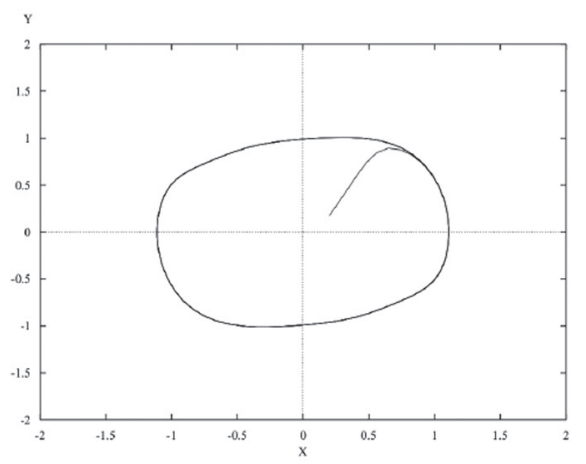

Figure 4. Example 3.6.

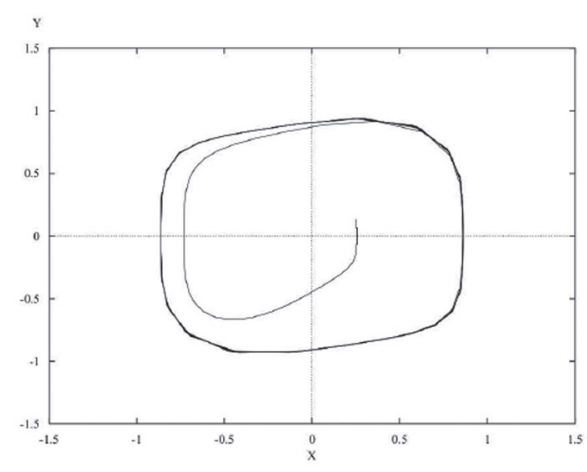

Figure 5. Example 3.7.

Our existence results raise some natural problems which deserve greater understanding for example: to obtain conditions for uniqueness, bounds of the number of cycles, stability or hyperbolicity of the founded limit cycles. 


\section{Acknowledgement}

The authors would like to thank the anonymous referees for their interesting comments.

\section{References}

[1] Arrowsmith D.K. and Place C.M., Dynamical systems. Differential equations, maps and chaotic behaviour, Chapman \& Hall, London, 1992.

[2] Bendixson I., "Sur les curbes définiés par des équations différentielles" (French), Acta Math. 24 (1901), No. 1, 1-88.

[3] Carletti T., Rosati L. and Villari G., "Qualitative analysis of phase portrait for a class of planar vector fields via the comparison method", Nonlinear Anal. 67 (2007), No. 1, 39-51.

[4] Ciambellotti L., "Uniqueness of limit cycles for Liénard systems. A generalization of Massera's theorem", Qual. Theory Dyn. Syst. 7 (2009), No. 2, 405-410.

[5] Perko L., Differential equations and dynamical systems, Third edition, Texts in Applied Mathematics, 7, Springer-Verlag, New York, 2001.

[6] Poincaré H., "Mémoire sur les curbes définiés par une équation différentielle II", J. Math. Pures Appl. 8 (1882), 251-296.

[7] Ye Y.Q., Cai S.L., Chen L.S., Huang K.C., Lou D.J., Ma Z.E., Wang E.N., Wang M.S. and Yang X.A., Theory of limit cycles. Translations of Mathematical Monographs, 66, American Mathematical Society, Providence, RI, 1986. 